Reprod. Nutr. Dévelop., 1986, 26 (1 B), 147-159.

\title{
Interactions entre espèces microbiennes anaérobies dans le rumen
}

\author{
Ph. GOUet, J. GRAIN $(*)$, H. C. DUBOURGUIER $(* *)$, G. ALBAGNAC $(* *)$ \\ Laboratoire de Microbiologie, I.N.R.A., Theix, 63122 Ceyrat France. \\ (*) Laboratoire de Biologie comparée des Protistes, LA 138 CNRS, \\ Université de Clermont II, 63170 Aubière. \\ (**) Station de Technologie alimentaire, I.N.R.A., \\ 59651 Villeneuve d'Ascq.
}

Summary. Interactions between species of anaerobic microbes in the rumen.

The rumen is a strictly anaerobic ecosystem colonized by an extremely dense microflora and microfauna. These populations are composed of a grand variety of bacterial and protozoal species. Their cohabitation allows observation of most of the known stages of communal life. The different hydrolytic, fermentative and methanogenic activities of these populations ensure the efficient degradation of cell wall constituent in forages (cellulose, hemicellulose, pectin) ingested by ruminants.

\section{Introduction.}

Le rumen, principal réservoir digestif des ruminants, constitue un écosystème anaérobie strict où la plupart des constituants des aliments sont dégradés et fermentés par une microflore et une microfaune extrêmement nombreuses et diversifiées. La population bactérienne, dont la concentration s'élève à $10^{10}-10^{11}$ cellules $\mathrm{ml}^{-1}$, représente la moitié de la masse microbienne et la catégorie de microorganismes la plus variée. La microfaune est composée essentiellement de ciliés $\left(10^{6} \mathrm{ml}^{-1}\right)$ dont le rôle est moins connu que celui des bactéries en raison des difficultés à les cultiver in vitro (Coleman, 1978). Enfin des champignons anaérobies stricts cellulolytiques (Bauchop, 1981) apparaissent avec certains régimes contenant des fourrages grossiers, mais leur fonction n'a pas encore été élucidée. Les aliments qui servent de substrat à la croissance microbienne sont principalement des fourrages riches en constituants pariétaux (cellulose, hémicelluloses, pectines), des sous-produits industriels (pailles, déchets ligno-cellulosiques); on leur associe fréquemment diverses formes d'azote non protéique (urée) dont I'ammoniaque.

Les différentes espèces du rumen sont spécialisées dans des fonctions métaboliques parfois limitées mais leur diversité leur permet de se recouvrir largement et le cas échéant, de se substituer les unes aux autres, l'ensemble constituant une chaîne trophique. Ainsi tout au long de l'évolution, au cours de millions d'années, 
ces espèces microbiennes se sont progressivement adaptées à cœxister dans cet écosystème en développant des interactions multiples et complexes (Russel et Hespell, 1981) qui lui confèrent une stabilité et une efficacité remarquables.

On trouve dans le rumen tous les genres de vie communautaire (Meers, 1973) : la " compétition » lorsque deux espèces dépendent du même substrat limitant, le " neutralisme » lorsque deux espèces coexistent sans influences réciproques, le " commensalisme » si la croissance de l'une dépend de l'autre, sans que cette dernière soit influencée par la première, le "syntrophisme " qui implique un transfert d'hydrogène obligatoire à partir de souches hydrogénophiles ; la production de substances toxiques amène aux cas d' "amensalisme " et enfin la " prédation » est fréquente chez les protozoaires qui ingèrent des bactéries.

Les mécanismes de ces interventions peuvent être d'ordre physiologique, nutritionnel ou métabolique mais nos connaissances actuelles sont encore trop limitées pour qu'il soit possible de hiérarchiser leur importance respective.

\section{Interactions entre espèces bactériennes}

\section{Chaînes trophiques dans le rumen.}

On peut distinguer schématiquement trois groupes microbiens selon la nature et la complexité du substrat énergétique utilisé pour leur croissance, certaines espèces pouvant appartenir aux deux premiers groupes simultanément.

Le groupe des "hydrolytiques " comprend principalement des bactéries (Bacteroides succinogenes, Ruminococcus albus, Ruminococcus flavefaciens, Butyrivibrio fibrisolvens, Streptococcus bovis, ...) mais aussi des protozoaires et même des champignons (Amos et Akin, 1978, Cheng, Dindsdale et Stewart, 1979) ; leurs cellules se fixent généralement aux fibres et dépolymérisent les polysaccharides pariétaux ou l'amidon en oses simples (cellobiose, maltose, saccharose, xylobiose, ...l.

Ces oses sont ensuite fermentés par les espèces du groupe "fermentatif 》 en acides gras dont les principaux sont l'acétate, le propionate, le butyrate, auxquels il faut ajouter du gaz carbonique et de l'hydrogène. De plus, des métabolites fermentaires comme l'éthanol, le succinate et le lactate qui sont produits en culture pure par plusieurs espèces, ne s'accumulent pourtant pas dans le rumen car ils sont catabolisés par d'autres au fur et à mesure de leur apparition. Le gaz carbonique est réduit par l'hydrogène en méthane par le groupe des méthanogènes. Ceux-ci jouent un rôle déterminant dans la cellulolyse en transférant l'hydrogène dont I'accumulation inhibe certaines espèces de cellulolytiques.

Enfin un troisième groupe réunissant plusieurs espèces des premier et second groupes (B. fibrisolvens, S. bovis, B. amylophilus, Bacteroides ruminicola, Selenomonas ruminantium) peuvent dégrader les substrats azotés pour former outre l'acétate déjà produit par ailleurs, des acides gras ramifiés et de l'ammoniaque indispensable à la croissance de nombreuses espèces. 


\section{Compétition pour le substrat.}

Parmi tous les mécanismes qui limitent la croissance bactérienne et contrôlent les équilibres régnant entre des dizaines d'espèces différentes, la compétition pour un substrat limitant se situe probablement aux tout premiers rangs. La domination d'une espèce sur les autres dépendra de ses besoins en énergie, azote et nutriments essentiels ainsi que de leur disponibilité dans le milieu et du pH (Russel et Baldwin, 1979). Mais outre la présence indispensable de ces sources, la vitesse et l'efficacité de leur utilisation sont aussi essentielles. Des populations bactériennes en compétition pourront donc coexister aussi longtemps que des substrats limitants différents seront présents dans le milieu, chacun d'eux favorisant la population qui l'utilisera le plus efficacement.

Avec des régimes riches en céréales $S$. bovis, espèce amylolytique, devient dominant. II produit des concentrations élevées d'acide lactique ce qui entraîne l'abaissement du $\mathrm{pH}$ puis la régression de plusieurs espèces parmi lesquelles se trouvent les cellulolytiques et des espèces qui fermentent le lactate, à l'exception de Megasphera elsdenii qui émerge rapidement (Counotte et al., 1981). La domination de $S$. bovis ne peut pas être expliquée par ses seules aptitudes amylolytiques car de nombreuses bactéries du rumen utilisent I'amidon aussi bien. II semble que l'avantage soit plutôt dû à son taux de croissance très élevé, associé à une diminution rapide du pH (Russel, Sharp et Baldwin, 1979) jusqu'à un niveau où les taux de croissance de la plupart des autres espèces sont ralentis (Therion, Kistner et Kornelius, 1982). Cette explication globale ne doit pas masquer l'extrême complexité du phénomène. La co-culture de $S$. bovis et $M$. elsdenii avec de l'amidon comme seule source énergétique, mais en faisant varier le taux de dilution, montre que la compétition entre ces deux espèces est influencée par plusieurs paramètres physiologiques tels que leur affinité pour le maltose, leur taux de croissance maximum, leur énergie de maintenance, leur rendement, la production et l'utilisation du lactate et enfin leur résistance aux faibles $\mathrm{pH}$ (Russel et Allen, 1984).

\section{Détoxification du milieu.}

Le rumen est un ècosystème anaérobie bien que des quantités significatives d'oxygène puissent y être détectées (jusqu'à 1,6 $\mu \mathrm{mol} \mathrm{O}_{2}$ ) (Scott et al., 1983). Très rapidement après les repas, l'oxygène dissous redescend à des niveaux non mesurables, grâce à sa consommation lors du catabolisme des sucres facilement fermentescibles par des bactéries anaérobies facultatives présentes dans la flore du rumen (Hungate, 1966). L'apport de glucose à du jus de rumen oxygéné se traduit par une augmentation transitoire de la pression partielle d'hydrogène et de la vitesse de consommation de l'oxygène, ce qui témoigne de l'activité métabolique des bactéries anaérobies facultatives et de l'inhibition de la méthanogénèse par l'oxygène (Scott et al., 1983). Ainsi les bactéries fermentatives doivent intervenir dans le maintien d'un bas potentiel redox, indispensable à la stabilité de l'écosystème même en présence d'entrées d'oxygène lors de la rumination ou de la prise d'aliments. 
Les fourrages consommés par les ruminants contiennent communément des acides phénoliques tels que les acides cinnamiques (acides férulique et $p$ coumarique) et surtout les acides hydroxycinnamiques. Ces acides peuvent aussi être présents sous forme soluble dans les pailles de céréales traitées par des alcalis (Salomonsson, Theander et Man, 1978). Les acides férulique et $p$-coumarique semblent être les plus toxiques car à la concentration de $5 \mathrm{mM}$ ils inhibent sur certains milieux la croissance de $R$. albus, $R$. flavefaciens, $B$. succinogenes et sur d'autres milieux ils réduisent l'activité cellulolytique de $R$. albus et $R$. flavefaciens. Toutefois la réduction de ces acides par la flore du rumen en acides phénylpropioniques permet de lever in vitro l'inhibition de la croissance et de l'activité cellulolytique (Chesson, Stewart et Wallace, 1982).

\section{Commensalisme et interactions nutritionnel/es.}

L'apport d'une ration finement broyée ou contenant des sucres facilement fermentescibles produit une baisse de $\mathrm{pH}$ et de la production d'acétate et une augmentation de la concentration en propionate. Ce dernier peut provenir du lactate produit par $S$. bovis et certains Lactobacillus et dans ce cas, la voie utilisée est celle de l'acrylate $(M$. elsdenii). Cependant l'essentiel du propionate est dérivé du succinate (Blackburn et Hungate, 1963) qui est décarboxylé par $S$. ruminantium. Parmi les principales espèces bactériennes amylolytiques et lactilytiques, la sensibilité de $B$. fibrisolvens au $\mathrm{pH}$ est suffisante pour expliquer son élimination de la flore dominante du rumen chez les animaux recevant des régimes riches en amidon (Therion, Kistner et Kornelius, 1982). De même, bien que le formate soit un produit courant de la fermentation des glucides, il n'est jamais trouvé dans le rumen car il est très rapidement utilisé et métabolisé en méthane par Methanobrevibacter ruminantium.

De nombreuses espèces dominantes dans le rumen possèdent in vitro des exigences nutritionnelles particulières généralement couvertes par l'addition de jus de rumen stérile aux milieux de culture. Ainsi Succinivibrio fibriso/vens est dépendant pour la 1-4 naphtoquinone (Gomez-Alarcon et al., 1982) et $M$. ruminantium pour l'acide 2-méthyl-butyrique, le coenzyme $M$ et certains acides aminés (Bryant et al., 1971 ; Taylor et al., 1974). Ce dernier exemple illustre bien les interactions qui peuvent exister entre les bactéries qui fermentent les composés azotés et les autres bactéries de l'écosystème par l'intermédiaire des acides gras ramifiés. En outre les bactéries qui dégradent les acides aminés ou l'urée sont à l'origine de l'ammoniaque et contribuent à ce titre au maintien du $\mathrm{pH}$ et à la nutrition azotée de la plupart des bactéries de l'écosystème. Enfin la plupart des bactéries anaérobies présentent une meilleure vitesse de croissance en présence de $\mathrm{CO}_{2}$. Ainsi chez $B$. fibrisolvens, l'augmentation de la concentration en bicarbonate dans le milieu augmente le rendement de croissance sur glucose et la production de formate (Jarvis, 1978).

\section{Transferts interspécifiques d'hydrogène.}

Les bactéries méthanogènes hydrogénophiles ont pour effet d'éliminer en permanence du milieu l'hydrogène toxique pour certaines souches de bactéries hydrolytiques, telles que Clostridium cellobioparum (Chung, 1976) et de contri- 
buer au maintien du pH en favorisant le déplacement de l'équilibre des réactions de production d'hydrogène et donc de réduction des protons. Les transferts interspécifiques d'hydrogène dans le rumen sont maintenant reconnus comme le facteur le plus important régulant les profils fermentaires (Hespell, 1981). La vitesse spécifique de consommation d'hydrogène par la population bactérienne est relativement élevée (13 à $30 \mathrm{mM} . \mathrm{I}^{-1} \cdot \mathrm{h}^{-1}$ ) (Robinson et Tiedje, 1982) et dans les conditions normales, la pression partielle de $\mathrm{H}_{2}$ est de l'ordre de $10^{-4}$ atmosphère (Hungate, 1966). La production d'hydrogène à partir des coenzymes NADH ou NADPH devient dans ces conditions thermodynamiquement possible (Wolin, 1974). Cela permet une déviation presque complète du métabolisme des bactéries hydrolytiques ou fermentatives vers la production de 2 moles d'acétate par mole de glucose fermenté et donc un gain net d'énergie. Deux exemples ont été bien étudiés in vitro. Chez $R$. flavefaciens associé à $M$. ruminantium (Wolin, 1974) la production de succinate et de formate est extrêmement réduite au profit de celle d'acétate. Dans le cas de $R$. albus, I'association avec Wolinella succinogenes se traduit par la disparition totale de la production d'éthanol et d'hydrogène (lanotti et al., 1973). Ce type de déviation a été récemment mis en évidence entre les champignons anaérobies cellulolytiques du rumen et les méthanogènes hydrogénophiles (Bauchop et Mountfort, 1981). Très généralement, ces transferts interspécifiques d'hydrogène inhibent la production de composés réduits et dévient le métabolisme vers une production accrue d'acétate, d'où une augmentation nette de la synthèse d'ATP et des vitesses de croissance de la flore fermentative.

Les écosystèmes anaérobies strictes méthanogènes sont seuls à présenter un cas d'interaction tout à fait original, la syntrophie, qui implique un transfert interspécifique d'hydrogène obligatoire. Quelle que soit la composition du substrat, une espèce syntrophe ne peut se développer sans la présence d'une hydrogénophile (méthanogène ou sulfato-réductrice), qui en maintenant une pression partielle d'hydrogène très basse rend possible au plan thermodynamique la dégradation de certains composés comme les alcools et les acides gras ; de même la dégradation anaérobie par le jus de rumen in vitro des noyaux benzéniques mono- ou disubstitués des acides phénoliques, constituants unitaires de la lignine (Chesson, Stewart, Wallace, 1982), est largement défavorable dans les conditions standard et nécessite donc un transfert interspécifique d'hydrogène. Dans le syntrophisme aucun moyen chimique ou physique actuellement connu ne peut se substituer au mécanisme biologique ce qui le différencie notoirement du commensalisme. Plusieurs types de bactéries syntrophes ont été décrites : Syntrophobacter wolinii qui réalise l'acétogénèse à partir du propionate (Boone et Bryant, 1980), Syntrophomonas wolfii dégradant le butyrate (Mc Inerney et al., 1981) et Syntrophus buswelli (Mountfort et al., 1984) catabolisant le benzoate.

Enfin, les lipides sont hydrolysés dans le rumen, le glycérol étant fermenté en acétate, propionate et succinate par Anaerovibrio lipolytica (Hobson et Mann, 1961). La dégradation anaérobie de ces acides gras relargués peut être réalisée en présence de sulfate par des bactéries sulfato-réductrices des genres Desulfosarcina, Desulfonema et Desulfococcus (Widdel et Pfennig, 1984), mais celles-ci sont incapables de jouer le rôle de syntrophes. Actuellement des cultures syntrophiques non sulfato-réductrices (Roy, Samain et Albagnac, 1985) capables de 
dégrader des acides à longues chaînes (palmitate, oléate, stéarate) ont été obtenues et sont en cours de caractérisation.

\section{Interactions entre bactéries et protozoaires}

\section{Influence de la microfaune sur les fermentations.}

Les ciliés du rumen représentent une biomasse qui peut, avec certains régimes, égaler celle des bactéries. Cinq à six genres différents, Entodinium, Polyplastron, Isotricha, Epidinium, Eudiplodinium, sont généralement présents simultanément mais les associations rencontrées sont très variées. Cette diversité peut être due à la répartition géographique des hôtes et à la nature des aliments (Grain, 1967). Si leur importance vis-à-vis de l'hôte est controversée dans la mesure où ils ne sont pas nécessaires à la digestion, ils fournissent par contre de nombreux exemples d'interactions entre eux ou avec les bactéries, ce qui rend leur étude particulièrement intéressante. Malheureusement les mécanismes de ces interactions sont le plus souvent inconnus en raison des difficultés à cultiver les ciliés in vitro. D'une manière générale la défaunation se répercute directement sur les caractéristiques fermentaires du rumen en modifiant les proportions des différents acides gras volatils, les productions d'ammoniaque (Jouany, 1978) et de méthane (Demeyer, 1981). La digestibilité de la matière organique et de la lignocellulose est plus faible chez les moutons défaunés quel que soit le régime (Grolière et al., 1978 ; Jouany et al., 1981 ; Jouany et Sénaud, 1983) mais le pH est plus élevé. Ceci va de pair avec une plus faible concentration d'acides gras volatils totaux et indique que les ciliés favorisent la digestion des glucides dans le rumen soit par action directe, soit en stimulant le métabolisme des bactéries, soit en combinant ces deux actions (Jouany et Sénaud, 1983). Enfin, la quantité de cellulose dégradée par les bactéries cellulolytiques diminue quand on implante Polyplastron avec un régime pauvre en cellulose, en raison peut-être, de la compétition pour le substrat (Jouany et Sénaud, 1982).

Ces modifications des fermentations liées à la défaunation et (ou) la faunation sont probablement en rapport avec des variations quantitatives des populations bactériennes. Même si les techniques de dénombrement bactérien gagnent à être affinées, il semble que le nombre de bactéries diminue lors de la faunation ; la microflore serait ainsi réduite de 50 à $75 \%$ chez des agneaux à flores limitées après l'introduction de Entodinium ou de Polyplastron en raison peut-être de la prédation des ciliés sur les bactéries (Fonty et al., 1983). Enfin, la répartition des catégories de bactéries en tailles et classes métaboliques serait modifiée (Jouany et al., 1981).

\section{Interactions dues à l'implantation des protozoaires.}

Les conditions naturelles d'implantation et de développement des ciliés témoignent tout particulièrement de leur dépendance vis-à-vis de l'environnement bactérien propre à l'écosystème rumen. Ainsi Entodinium et Polyplastron ne survivent que quelques jours en fermenteur en l'absence de bactéries anaérobies stric- 
tes (Bonhomme, Fonty et Sénaud, 1982). Les ciliés apparaissent dans le rumen plus tardivement que les bactéries, au cours de la seconde semaine qui suit la naissance quand I'hôte commence à ingérer des aliments solides (Fonty et al., 1984) et la composition de ces derniers agit sur la vitesse d'installation et sur les effectifs au maximum du plateau (Grain et al., 1980 ; Collombier, 1981). Entodinium apparaît toujours le premier, suivi par les gros entodiniomorphes.

Après son apparition la population des ciliés croît pendant 15 à 20 jours pour atteindre des valeurs maximales vers 60 jours, mais au cours du troisième mois, les ciliés disparaissent totalement pendant 1 à 4 semaines chez $60 \%$ des agneaux élevés en troupeau. Cette défaunation naturelle affecte surtout Polyplastron qui disparaît chez $90 \%$ des individus pour des causes qui ne sont pas encore connues et la faunation qui suit ne conduit pas toujours à l'établissement des mêmes espèces (Fonty et al., 1984).

Des ciliés des genres Entodinium, Polyplastron et /sotricha ont été implantés séparément ou conjointement dans des rumens défaunés ou contenant déjà un ou deux des trois genres et chez des animaux recevant des régimes alimentaires différents (Sénaud et al., 1980 ; Grolière et al., 1980 ; Grain et al., 1980 ; Fonty et al., 1984). Dans les cas d'inoculation d'une faune mixte contenant Entodinium c'est toujours ce genre qui a proliféré le premier, l'ordre de développement des autres genres dépendant de la composition de l'inoculum. D'une manière générale, les populations implantées croissent rapidement pour atteindre un effectif maximum entre 5 et 14 jours.

La présence préalable d'autres genres de ciliés ou l'implantation simultanée de plusieurs genres dans le rumen peut modifier les vitesses d'installation et les effectifs. Par exemple, sur foin seul, Polyplastron s'établit plus vite s'il est implanté après Entodinium déjà établi depuis 5 jours ou conjointement avec Entodinium, que s'il est implanté seul dans un rumen défauné depuis 10 jours. L'établissement de Polyplastron et Entodinium ne peut être obtenu qu'avec une flore très diversifiée puisque 30 souches bactériennes représentant les principales espèces du rumen de l'adulte se sont révélées insuffisantes à cet égard chez des agneaux gnotoxéniques (Fonty et al., 1983 ; Fonty, 1984).

\section{Ingestion des bactéries.}

Bien que tous les ciliés du rumen avalent et digèrent des bactéries, on a cependant très peu de renseignements sur les quantités ingérées et sur l'éventualité d'un choix à l'ingestion ou d'une digestion sélective. Entodinium caudatum, élevé in vitro avec Klebsiella sp. et Proteus sp. contient environ 22 bactéries $( \pm 8)$ par cellule (White, 1969) et le même cilié, prélevé in vivo en contient $11( \pm 12)$; celles-ci sont surtout des Veillonella sp., Butyrivibrio sp., Streptococcus bovis (Coleman et White, 1970). Epidinium et Entodinium avalent une grande variété de bactéries mais assez lentement. Polyplastron avale plus rapidement un grand nombre de bactéries (de 1500 à 137 000/h/cellule) ; il n'effectue pas de tri, mais ingère certaines espèces plus que d'autres. II faut toutefois remarquer que Proteus mirabilis, qui n'est pas une bactérie du rumen, figure parmi les plus consommées (Coleman et Laurie, 1977). 
Les bactéries fixées aux particules végétales ou incluses dans les cellules végétales dégradées, sont avalées avec le végétal par les ciliés. Chez Epidinium, il est net que ces bactéries sont isolées par le cilié pendant l'ingestion, dans des vacuoles particulières où elles sont dégradées. Beaucoup de ces bactéries sont expulsées, intactes, par le cytoprocte du cilié (Grain et Sénaud, 1984).

\section{Interactions entre protozoaires}

Eadie (1962a, b, 1967), Sénaud et al. (1980) ont montré que les relations entre les genres de ciliés dans le rumen peuvent être définies soit comme un antagonisme, soit comme une neutralité. Avec des régimes variés (foin seul, concentrés ou mixte), Eadie (1962a) a mis en évidence deux types de populations chez des animaux à faune totale : I'une constituée par Entodinium, Isotricha, Dasytricha, des ciliés du groupe A (Polyplastron, Diplodinium affine, Ophryoscolex), l'autre composée de Entodinium, Isotricha, Dasytricha et des ciliés du groupe B (Eudiplodinium maggii, Epidinium). Les inoculums d'un mélange des deux types sont instables et tendent vers le premier type qui est prédominant chez le mouton (Eadie, 1962b). L'antagonisme entre A et B dont on ne connaît pas la nature, n'implique pas Entodinium, Isotricha et Dasytricha. En outre plusieurs auteurs ont montré que Polyplastron est capable de prédation vis-à-vis des autres ciliés. De travaux plus récents (Sénaud et al., 1980 ; Grolière et al., 1980) il ressort qu'il est impossible de dégager des lois générales sur les relations entre espèces de ciliés. Selon les régimes et les espèces en présence, les relations peuvent être très différentes et certaines compétitions qui n'ont pas été observées peuvent apparaître entre des espèces qui semblaient non compétitives.

Chez des moutons adultes fistulés, initialement défaunés, on observe entre Isotricha et Polyplastron, avec un régime riche en amidon (luzerne 40 - orge 50), une légère compétition pour l'amidon qui se traduit par une baisse de l'effectif de l'un des deux genres quand l'autre est introduit (fig. 1a, 1b) ; la prédation ne joue pas car Polyplastron n'ingère pas /sotricha. Cette relation entre les deux genres n'a pu être étudiée que dans ce cas car /sotricha ne s'installe pas en l'absence d'amidon dans la ration.

Entre Isotricha et Entodinium, avec des régimes contenant de l'amidon il existe une petite compétition pour cet aliment. S'il y a peu d'amidon, Isotricha l'utilise, alors qu'Entodinium consomme les fructosanes du foin ; si /sotricha est absent, alors Entodinium utilise d'abord l'amidon ; la compétition pour l'amidon tourne donc à l'avantage d'/sotricha, mais Entodinium subsiste parce qu'il s'adapte à un autre substrat.

Les relations entre Entodinium et Polyp/astron sont plus complexes. Avec un régime de foin seul sans amidon, il n'y a pas de compétition réelle. La présence d'Entodinium favorise le développement de Polyplastron qui manifeste une prédation modérée puisque Entodinium subsiste (fig. 1d). La présence de Polyplastron stimule ou active la prolifération de Entodinium, ce qui est une réponse à la prédation (fig. 1e, 1f). 
Avec un régime contenant $35 \%$ d'amidon, les deux genres ont un développement favorisé par l'amidon qu'ils consomment tous les deux; la prédation est faible et ils coexistent facilement.

\section{COMPETITIONS}
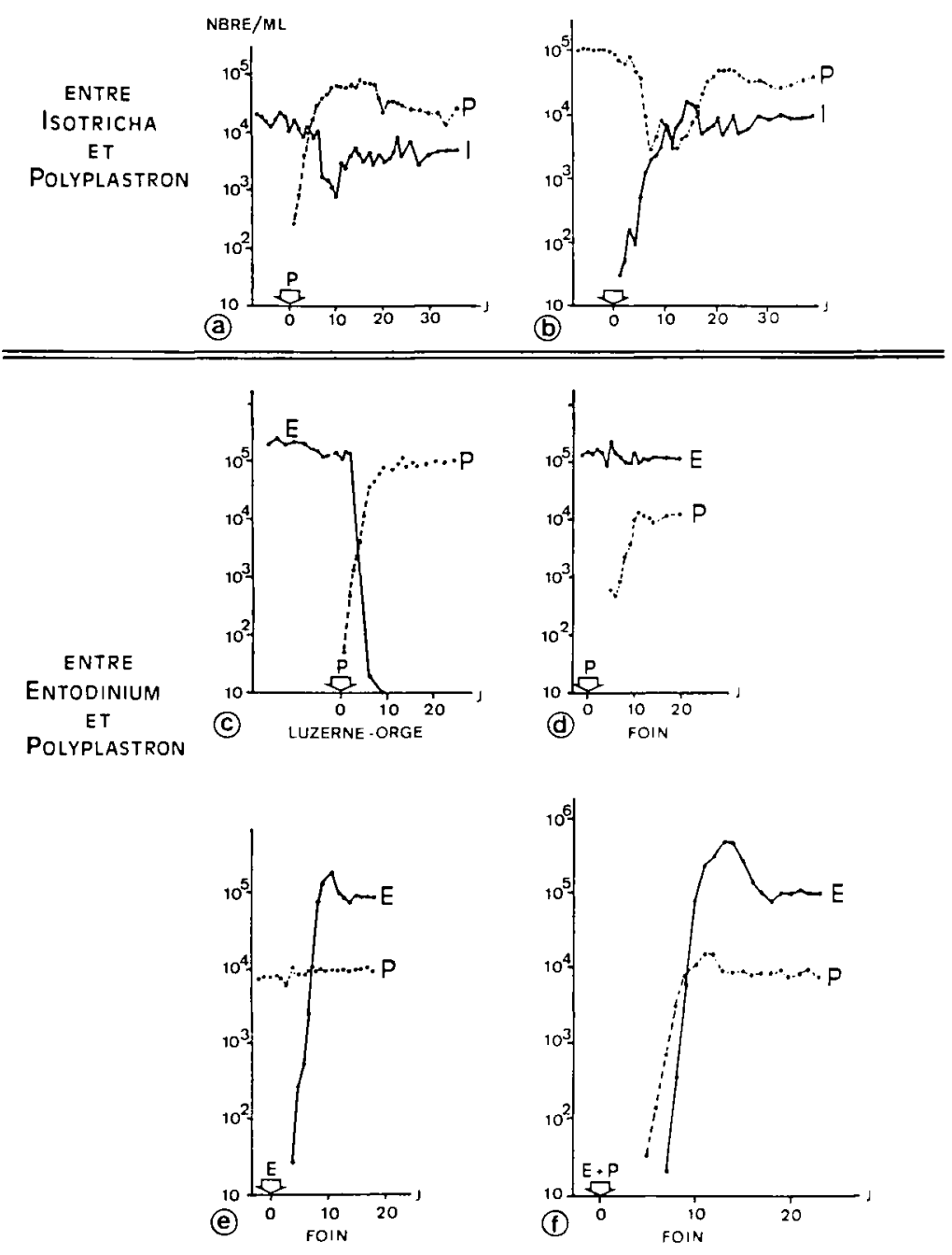

FIG. 1. - Compétitions entre divers genres de ciliés

$a$ et $b$ : entre /sotricha et Polyplastron avec régime luzerne-orge 40/50.

a : Implantation de Polyplastron après /sotricha au plateau (diminution de /sotricha) ; b : Implantation de /sotricha après Polyplastron au plateau (diminution de Polyplastron).

$c, d, e, f$ : entre Entodinium et Polyplastron.

c : régime luzerne-orge $40 / 50$ : implantation de Polyplastron après Entodinium au plateau ; diminution puis exclusion de Entodinium ; ${ }^{*} \mathrm{~d}$ : régime foin seul : implantation de Polyp/astron après Entodinium; * ${ }^{*}$ : régime foin seul : implantation de Entodinium après Polyplastron ; ${ }^{*} \mathrm{f}$ : régime foin seul : implantation simultanée de Entodinium et Polyplastron.

* = pas d'exclusion de Entodinium.

Reproduction, Nutrition, Développement, $n^{\circ} 1$ B-86. -3 
Avec un régime contenant entre 35 et $45 \%$ d'amidon, les deux genres consomment de l'amidon, mais cette compétition pour le substrat tourne au profit de Polyplastron. Il doit s'y ajouter une prédation importante et ces deux facteurs combinés font que si Polyplastron et Entodinium sont seuls en présence, la compétition aboutit à l'exclusion totale de Entodinium. Lorsque les deux genres sont présents simultanément au sein d'une faune totale, leurs relations sont différentes car Eudiplodinium et /sotricha également consommateurs d'amidon, sont en compétition pour le substrat avec Polyplastron. Ceux-ci, moins " activés " sans doute, changent de comportement vis-à-vis de Entodinium peut-être en raison d'une prédation plus faible. II est donc net, en particulier pour les relations entre Entodinium et Polyplastron, qu'un type de relation n'est valable que pour un régime précis.

Dans tous les cas étudiés, ce sont toujours les mêmes phénomènes qui sont invoqués : prédation, activation de la prolifération et compétition pour un aliment. La composition du régime alimentaire, la nature des genres en présence et la complexité de la faune sont autant de facteurs qui modulent l'intensité de ces phénomènes.

\section{Conclusions}

La variété des substrats dégradés dans le rumen, la multiplicité des espèces présentes, font de cet écosystème une source infinie d'interactions dont la connaissance devrait largement profiter aux autres écosystèmes anaérobies et particulièrement aux écosystèmes digestifs des monogastriques. En raison de la complexité de ces interactions très peu de mécanismes ont pu être mis en évidence en particulier pour les Protozoaires. De plus la nécessité de modéliser avec des associations simplifiées aboutit à ne dégager que des mécanismes d'interactions généraux issus de résultats in vitro qui ne tiennent compte, ni de l'absorption des métabolites par la muqueuse ruminale, ni de la répartition des bactéries. bactéries.

Les flux des populations du rumen restent très mal connus faute de techniques qui permettent de les différencier quantitativement. On peut espérer à moyen terme que la mise au point de techniques immuno-enzymatiques ou d'immunofluorescence pourront combler partiellement cette lacune pour des groupes aussi importants que les méthanogènes ou les cellulolytiques.

De plus, hous ne connaissons pas l'intensité des échanges inter-espèces et seules des techniques mettant en jeu des traceurs isotopiques radio-actifs $\left({ }^{14} \mathrm{C}\right)$ ou stables $\left({ }^{13} \mathrm{C},{ }^{2} \mathrm{D},{ }^{15} \mathrm{~N}\right)$ pourront nous informer sur les flux de carbone, d'azote ou d'hydrogène en termes quantitatifs.

Les techniques de mesure de la pression partielle d'hydrogène ou de détermination des pools des divers métabolites dans le liquide interstitiel ne renseignent que très globalement sur les conditions physiologiques régnant dans le rumen et nullement sur les phénomènes tels qu'ils doivent exister au niveau cellulaire dans les communautés bactériennes fixées aux fibres ou à la muqueuse. Les résultats énergétiques et métaboliques obtenus in vitro sont donc à considérer avec pré- 
caution et ne peuvent certainement pas être extrapolés sans réserves aux populations complexes.

Dans le cas spécifique du rumen, les influences liées à la physiologie de I'hôte peuvent modifier sensiblement le fonctionnement des populations bactériennes ; le ruminant gnotoxénique représente, à ce tirre, un apport expérimental capital pour l'étude in vivo de systèmes bactériens simplifiés (Fonty, 1984).

7ères Journées sur la Nutrition et l'Alimentation des Herbivores, I.N.R.A., Paris, 21 et 22 mars 1985.

\section{Références}

AMOS H. E., AKIN D. E., 1978. Rumen protozoal degradation of structurally intact forage tissues. Appl. environ. Microbiol., 36, 513.

BAUCHOP T., 1981. The anaerobic fungi in rumen fibre digestion. Agr. Environ., 6, 339-348.

BAUCHOP T., MOUNTFORT D. O., 1981. Cellulose fermentation by a rumen anaerobic fungus in both the absence and the presence of rumen methanogens. Appl. environ. Microbiol., 42, 1103-1110.

BLACKBURN T. H., HUNGATE R. E., 1963. Succinic acid turnover and propionate production in the rumen. Appl. Microbiol., 11, 132-135.

BONHOMME A., FONTY G., SENAUD J., 1982. Obtention de Polyplastron multivesiculatum (cilié entodiniomorphe du rumen) en condition axénique. J. Protozool., 29, 231-233.

BOONE D. R., BRYANT M. P., 1980. Propionate degrading bacterium, Syntrophobacter wolini from methanogenic ecosystems. Appl. environ. Microbiol., 40, 626-632.

BRYANT M. P., TZENG S. F., ROBINSON I. M., JOYNER A. E., 1971. Nutrients requirements of methanogenic bacteria. Adv. Chem. Ser., 23, 105-108.

CHESSON A., STEWART C. S., WALLACE R. J., 1982. Influence of plant phenolic acids on rumen bacteria. Appl. environ. Microbiol., 44, 597-603.

CHENG K. J., DINDSDALE D., STEWART C. S., 1979. Maceration of clover and grass leaves by Lachnospira multiparus., Appl. environ. Microbiol., 38, 723.

CHUNG K. T., 1976. Inhibitory effect of $\mathrm{H}_{2}$ on growth of Clostridium cellobioparum. Appl. environ. Microbiol., 31, 342-348.

COLEMAN G. A., 1978. Rumen entodiniomorphid protozoa, 39-54. In A. E. TAYLOR, J. BACKER, Method of cultivating parasites in vitro, Acad. Press.

COLEMAN G. S., LAURIE J. 1., 1977. The metabolism of starch, glucose, amino acids, purines, pyrimidines and bacteria by the rumen ciliate Polyplastron multivesiculatum. J. gen. Microbiol., 98, 29-37.

COLEMAN G. S., WHITE R. S., 1970. Re-establishment of Entodinium caudatum cultured in vitro, in the rumen of a defaunated sheep. J. gen. Microbiol., 62, 265-266.

COLLOMBIER J., 1981. Contribution à l'étude du rôle des protozoaires ciliés du rumen dans l'apport d'azote microbien entrant dans le duodénum du Ruminant. Th. Doct. Ing., Univ. Clermont II, p. 85.

COUNOTTE G. H. M., PRINS R. A., JANSSEN R. A. M., de BIE M. J. A., 1981. Role of Megasphaera e/sdenii in the fermentation of $\mathrm{DL}-\left(2-{ }^{13} \mathrm{C}\right)$ lactate in the rumen of dairy cattle. Appl. environ. Microbiol., 42, 649-655.

DEMEYER D. I., 1981. Rumen microbes and digestion of plant cell walls. Agric. Environ., 6. 295-337.

EADIE J. M., 1962a. The development of rumen microbial populations in lambs and calves under various conditions of management. J. gen. Microbiol., 29, 563-578.

EADIE J. M., 1962b. Inter-relationships between certain rumen ciliate protozoa. J. gen. Microbio/., 29, 579-588.

EADIE J. M., 1967. Studies on the ecology of certain rumen ciliate protozoa. J. gen. Microbiol., 49. 175-194. 
FONTY G., 1984. Implantation de la microflore et des Protozoaires ciliés dans le rumen d'agneaux holoxéniques et néoholoxéniques. Facteurs écologiques déterminant l'implantation de Bacteroides succinogenes (s85) et des protozoaires ciliés dans le rumen d'agneaux holoxéniques isolés d'agneaux méroxeniques et d'agneaux gnotoxéniques. Evolution des principaux paramètres digestifs. Th. Doct. ès Sci. nat., Univ. Clermont II, $\mathrm{n}^{\circ} \mathrm{E} 337$.

FONTY G., GOUET Ph., JOUANY J. P., SÉNAUD J., 1983. Ecological determining establishment of ceilulolytic bacteria and protozoa in the rumen of meroxenic lambs. J. gen. microbiol., 129, 213-223.

FONTY G., JOUANY J. P., SÉNAUD J., GOUET Ph., GRAIN J., 1984. The evolution of microflora, microfauna and digestion in the rumen of lambs from birth to 4 months. Canad. J. anim. Sci, 64 (suppl.), 165-166.

GOMEZ-ALARCON R. A., O'DOWD C., LEEDLE J. A. Z., BRYANT M. P., 1982. 1-4 Naphtoquinone and other nutrient requirements of Succinivibrio dextrinosolvens. Appl. environ. Microbiol., 44, 346-350.

GRAIN J., 1967. Le métabolisme des ciliés du rumen. Ann. Biol., 6, 17-45.

GRAIN J., SEENAUD J., 1984. New data on the degradation of fresh lucerne fragments by the rumen ciliate Epidinium ecaudatum attachment, ingestion and digestion. Can J. anim. Sci, 64 (suppl.) : 1-2, 26.

GRAIN J., SÉNAUD J., GROLIĖRE C. A., JOUANY J. P., 1980. Implantation et développement de Protozoaires ciliés (Polyplastron multivesiculatum, Entodinium sp., Isotricha prostoma) dans le rumen de moutons recevant différents régimes alimentaires. III. Considérations générales sur l'écosystème rumen. Protistologica, 16, 501-506.

GROLIĖRE C. A., SÉNAUD J., JOUANY J. P., GRAIN J., de PUYTORAC P., 1980. Implantation et développement de Protozoaires ciliés (Polyplastron multivesiculatum, Entodinium sp., Isotricha prostoma) dans le rumen de moutons recevant différents régimes alimentaires. II. Régimes à base de foin, enrichis en céréales. Protistologica, 16, 385-394.

GROLIĖRE C. A., SÉNAUD J., ZAINAB B., GRAIN J., JOUANY J. P., 1978. Rôle des ciliés dans la digestion d'une ration alimentaire luzerne-orge 40/50. 4th int. Congr. Parasitol., Varsovie, (Abstr.) sect. A, 26-27.

HESPELL R. B., 1981. Ruminal microorganisms. Their significance and nutritional value. In Dev. industr. Microbiol. Soc. indo. Micr., 22, 261-276.

HOBSON P. N., MANN S. O., 1961. Isolation of cellulolytic and lipolytic organisms from the rumen. J. gen. Microbiol., 25, 227-240.

HUNGATE R. E., 1966. The rumen and its microbes. Acad. Press., N.Y.

IANOTTI E. L., KAFFEWITZ D., WOLIN M. J., BRYANT M. P., 1973. Changes in glucose fermentation products of Ruminococcus albus grown in continuous culture with Vibrio succinogenes caused by interspecies transfer of $\mathrm{H}_{2}$. J. Bact., 114, 1231-1240.

JARVIS B. D. W., 1978. The role of carbonate in the metabolism of glucose by Butyrivibrio fibrisolvens. J. gen. Microbiol., 105, 287-295.

JOUANY J. P., 1978. Contribution à l'étude des protozoaires ciliés du rumen : leur dynamique, leur rôle dans la digestion et leur intérêt pour le Ruminant. Th. Doct. ès Sci. nat., Univ. Clermont II, $\mathrm{n}^{\circ} 256$.

JOUANY J. P., SÉNAUD J., 1982. Influence des ciliés du rumen sur la digestion de différents glucides chez le mouton. I. Utilisation des glucides pariétaux (cellulose et hémicellulose) et de l'amidon. Reprod. Nutr. Dévelop., 22, 735-752.

JOUANY J. P., SÉNAUD J., 1983. Influence des ciliés du rumen sur l'utilisation digestive de différents régimes riches en glucides solubles et sur les produits terminaux formés dans le rumen. II. Régimes contenant de l'insuline, du saccharose et du lactose. Reprod. Nutr. Dévelop., 23, 607-623.

JOUANY J. P., ZAINAB B., SÉNAUD J., GROLIĖRE C. A., GRAIN J., THIVEND P., 1981. Role of the rumen ciliate protozoa Polyplastron multivesiculatum, Entodinium sp. and Isotricha prostoma in the digestion of a mixed diet in sheep. Reprod. Nutr. Dévelop., 21, 871-884.

MC INERNEY M. J., BRYANT M. P., HESPELL R. B., COSTERTON J. M., 1981. Syntrophomonas wolfei, an anaerobic syntrophic fatty acid oxidizing bacterium. Appl. environ. Microbiol., 41, 1029-1039.

MEERS J. L., 1973. Growth of bacteria in mixed culture. Chem. Rubber Co. crit. Rev. Microbiol., 2, 139 . 
MOUNTFORT D. O., BRULLA W. J., KRUMHOLZ L. R., BRYANT M. P., 1984. Syntrophus bruswellii a benzoate catabolizer from methanogenic ecosystems., Int. J. syst. Bact., 34, 216217.

ROBINSON J. A., TIEDJE J. M., 1982. Kinetics of hydrogen consumption by rumen fluid, anaerobic digestor sludge and sediment. Applied environ. Microbiol., 43, 1374-1384.

ROY F., SAMAIN E., ALBAGNAC G., 1985. Influence of calcium addition on growth of highly purified syntrophic cultures degrading long-chain fatty acids. Appl. Microbiol., 49 (sous presse).

RUSSEL J. B., ALLEN M. S., 1984. Physiological basis for interactions among rumen bacteria : Streptococcus bovis and Megasphaera e/sdenii as a model, 239-247. In KLUG M. J., REDDY C. A., Current perspectives in microbial ecology, A.S.M. Washington D. C.

RUSSEL J. B., BALDWIN R. L., 1979. Comparison of substrate affinities among several rumen bacteria : a possible determinant of rumen bacterial competition. Appl. environ. Microbiol., 37, 531-536.

RUSSEL J. B., HESPELL R. B., 1981. Microbial rumen fermentation. J. Dairy Sci., 2, 513.

RUSSEL J. B., SHARP W. M., BALDWIN R. L., 1979. The effect of $\mathrm{pH}$ on maximum bacterial growth rate and its possible role as a determinant of bacterial competition in the rumen. $J$. anim. Sci., 48, 251-255.

SALOMONSSON A. C., THEANDER O., MAN P. A., 1978. Quantitative determination by GLC of phenolic acids as ethyl derivatives in cereal strain. J. agric. Food. Chem., 26, 1169-1175.

SCOTT R. I., YARLETT N., HILLMAN K., WILLIAMS T. N., WILLIAMS A. G., LLOYD D., 1983. The presence of oxygen in rumen liquor and its effects on methanogenesis. J. appl. Bact., 55, 143-149.

SÉNAUD J., GROLIËRE C. A., ZAINAB B., GRAIN J., JOUANY J. P., 1980. Implantation et développement des populations de Protozoaires ciliés (Polyplastron multivesiculatum, Entodinium sp., Isotricha protozoa) dans le rumen de moutons recevant différents régimes alimentaires. I. Régime luzerne-orge (40/50). Protistologica, 16, 329-337.

TAYLOR C. D., Mc BRIDE B. C., WOLFE R. S., BRYANT M. P., 1974. Coenzyme M essential for growth of a rumen strain of Methanobacterium ruminantium. J. Bact., 120, 974.

THERION J. J., KISTNER A., KORNELIUS J. H., 1982. Effect of $\mathrm{pH}$ on growth rates of rumen amylolytic and lactilytic bacteria. Appl. environ. Microbiol., 44, 428-434.

WIDDEL F., PFENNIG N., 1984. Dissimilatory sulfate or sulfur reducing bacteria. In KRIEG N. R. ed. Bergey's manual of systematic bacteriology, vol. 1, 9th Ed. Williams et Wilkins Publ., Baltimore.

WOLIN M. J., 1974. Interactions between $\mathrm{H}_{2}$-producing and methane producing species. In Microbial production and utilization of gases. Gottingen.

WHITE R. W., 1969. Viable bacteria inside the rumen ciliate Entodinium caudatum. J. gen. Microbiol., 56, 403-408. 